EPJ Web of Conferences 19, 01009 (2012)

DOI: $10.1051 /$ epjconf/20121901009

(C) Owned by the authors, published by EDP Sciences, 2012

\title{
The shape of dark matter haloes in the Aquarius simulations: Evolution and memory
}

\author{
C.A. Vera-Ciro ${ }^{1, a}$, L.V. Sales ${ }^{1,2}$ and A. Helmi ${ }^{1}$ \\ ${ }^{1}$ Kapteyn Astronomical Institute, Univ. of Groningen, P.O. Box 800, 9700 AV Groningen, \\ The Netherlands \\ ${ }^{2}$ Max-Plank-Institut für Astrophysik, Karl-Schwarzschild-Straße, 1, 85740 Garching bei \\ München, Germany
}

\begin{abstract}
We use the high resolution cosmological $N$-body simulations from the Aquarius project to investigate in detail the mechanisms that determine the shape of Milky Way-type dark matter haloes. We find that, when measured at the instantaneous virial radius, the shape of individual haloes changes with time, evolving from a typically prolate configuration at early stages to a more triaxial/oblate geometry at the present day. This evolution in halo shape correlates well with the distribution of the infalling material: prolate configurations arise when haloes are fed through narrow filaments, which characterizes the early epochs of halo assembly, whereas triaxial/oblate configurations result as the accretion turns more isotropic at later times. Interestingly, at redshift $z=0$, clear imprints of the past history of each halo are recorded in their shapes at different radii, which also exhibit a variation from prolate in the inner regions to triaxial/oblate in the outskirts. Provided that the Aquarius haloes are fair representatives of Milky Way-like $10^{12} \mathrm{M}_{\odot}$ objects, we conclude that the shape of such dark matter haloes is a complex, time-dependent property, with each radial shell retaining memory of the conditions at the time of collapse.
\end{abstract}

We use the Aquarius state of the art high resolution $N$-Body simulations of 5 Milky Way sized dark matter haloes (labeled Aq-A, .., Aq-E), in a $\Lambda \mathrm{CDM}$ universe. The haloes have a final mass of $\sim 10^{12}$ $\mathrm{M}_{\odot}$ and were selected to be relatively isolated at the present day [1].

The left panel of Fig. 1 shows the axis ratios as a function distance to the centre for each Aq-halo. The lengths $a \geq b \geq c$ are measured using an inertia tensor-based method [2]. We observe that in the inner regions all haloes are prolate $b \sim c$. Close the virial radius (vertical arrow) haloes become oblate/triaxial $b \sim a$. We have also found that the minor axis tend to point perpendicular to the direction of preferred infall which is in good agreement with previous works [3].

The central panel shows the evolution of the shape for the tidal contour, which is clearly a timedependent quantity. In general haloes evolve from prolate configurations at early times towards more triaxial shapes at present day. The larger scatter from halo to halo is due to the particular histories and local conditions. Interestingly, haloes show resemblance between the virial ellipsoid's shape with time and the shapes measured as a function of position.

To asses the importance of mass acretion on the onset of the shape at a given time, we characterize the accretion modes with a multipole expansion of the positions in the sky of particles in first infall (right panel in Fig. 1). We found that large values in the mode $\ell=2$ indicates the presence of net filamentary accretion, similarly the relative size halo/filament is nearly quantified by $\ell=0$. We reached to the conclusion that the early evolutionary phases of $10^{12} \mathrm{M}_{\odot}$ Milky Way-like haloes are characterized

\footnotetext{
${ }^{\mathrm{a}} \mathrm{e}$-mail: cavera@astro.rug.nl
}

This is an Open Access article distributed under the terms of the Creative Commons Attribution-Noncommercial License 3.0, which permits unrestricted use, distribution, and reproduction in any noncommercial medium, provided the original work is properly cited. 

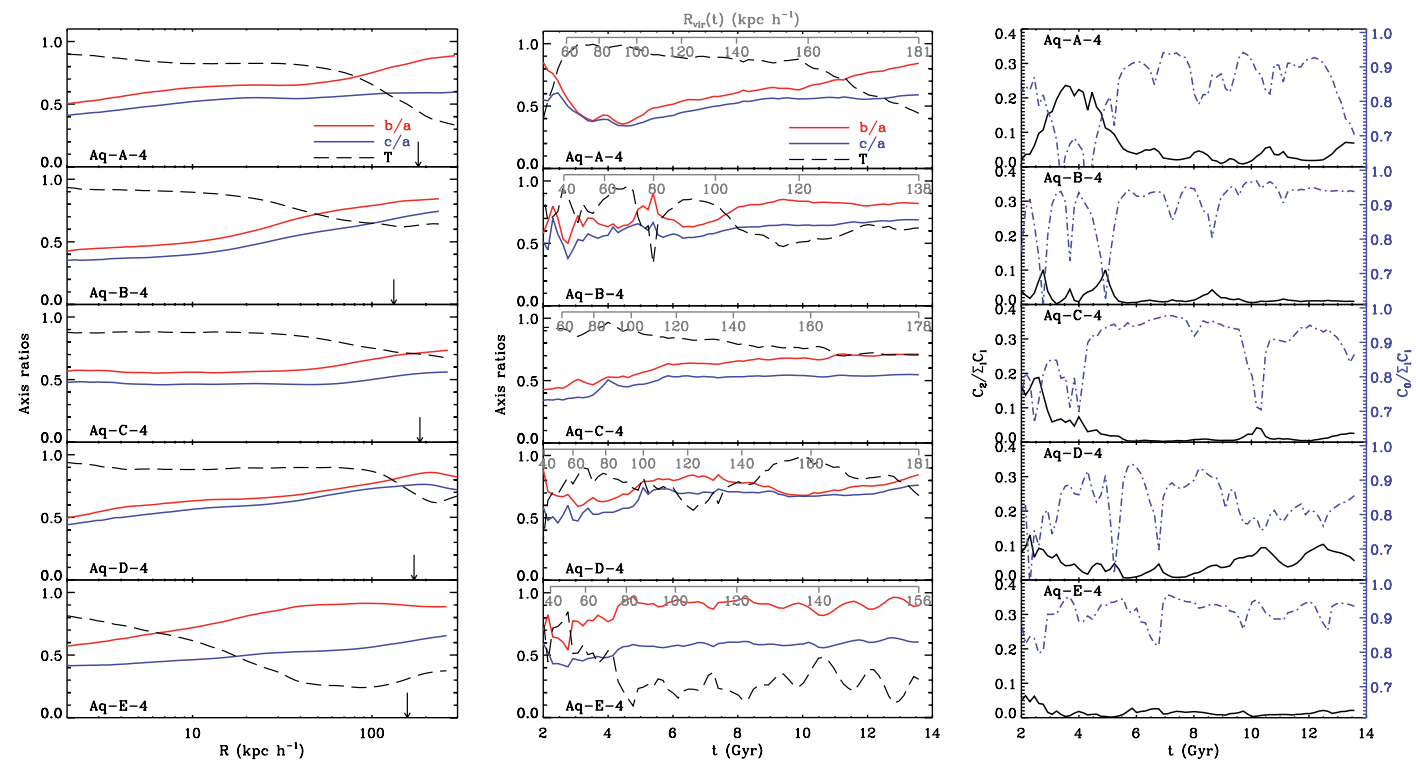

Figure 1. Axis ratios as a function of radius (left) and as a function of time for the virial contour (centre) for each of the Aq-haloes. In both panels the thick solid lines represent the axis ratios $b / a$ (red) and $c / a$ (blue), while the dashed curves are the triaxiality parameter $T=\left(a^{2}-b^{2}\right) /\left(a^{2}-c^{2}\right)$. In general haloes are more prolate in the inner parts and more oblate (and triaxial) in the outskirts. A comparison between these two figures shows that a correlation exists between the shape as a function of time, and the present-day shape as a function of radius. To aid this comparison we have added a second axis (gray), indicating the size of the virial contour $R_{\text {vir }}$ at each time. (right) Relative contribution of the $\ell=0$ (blue dot-dashed) and $\ell=2$ (black solid) modes to the total power spectrum as a function of time for particles on their first infall onto each halo. Notice the good correlation between the time intervals with a clear signature of mass accretion through filaments (high power in $\ell=2$ mode) and the more prolate shape.

by the accretion of matter through narrow filaments. In these circumstances the haloes -as measured by their virial contours - are prolate and their minor axes tend to point perpendicular to the infall (filament) direction. Nonetheless, temporary tilting of the virial ellipsoids may occur when mass is accreted from a different direction. The latter is the case for just one of our haloes located in a well defined filament at redshift $z=0$. On the other hand, at later times the cross-section of the filaments becomes larger than the typical size of Milky Way-like haloes and as a result, accretion turns more isotropic and the objects evolve into a more oblate/triaxial configuration. This transition does not occur at the same time for all haloes in the explored mass range but is strongly determined by their individual history of mass assembly and their surrounding environment. The geometrical properties of haloes at different epochs are not lost: haloes retain memory of their structure at earlier times. This memory is imprinted in their present-day shape trends with radius, which change from typically prolate in the inner (earlier collapsed) regions to a triaxial in the outskirts (corresponding to the shells that have collapsed last and are now at the virial radius) [4].

\section{References}

[1] Springel V. et al., MNRAS 391, (2008) 1685

[2] Allgood B. et al., MNRAS 367, (2006) 1781

[3] Bailin J., Steinmetz M., 2005, ApJ 627, (2005) 647

[4] Vera-Ciro C. et al., 2011, MNRAS 416, (2011) 1377 